\title{
Redes sociales como espacios comunicativos de articulación de movimientos sociales: Revolución de los Frijoleros (Guatemala)
}

Social media as communicative spaces for the articulation of social movements: Frijoleros Revolution (Guatemala)

Redes sociais como espaços comunicativos de articulação de movimentos sociais: Revolução dos Frijoleros (Guatemala)

\author{
Elizabet CASTILLERO-OSTIO \\ Universidad de Málaga (España) \\ ecastillero@uma.es \\ Marta GIL-RAMÍREZ \\ Universidad de Málaga (España) \\ martagr@uma.es

\section{Antonio CASTILLO-ESPARCIA \\ Universidad de Málaga (España) \\ acastilloe@uma.es}

\footnotetext{
Chasqui. Revista Latinoamericana de Comunicación

N. ${ }^{\circ}$ 148, diciembre 2021 - marzo 2022 (Sección Monográfico, pp. 67-88)

ISSN 1390-1079 / e-ISSN 1390-924X

Ecuador: CIESPAL

Recibido: 22-06-2021 / Aprobado: 12-11-2021
} 


\title{
Resumen
}

Esta investigación determina cuál es el papel que juegan las redes sociales en la organización del movimiento social durante la "Revolución de los Frijoleros" en Guatemala en 2020. A través de un enfoque mixto que combina el análisis de contenido y el análisis crítico del discurso, se analizan cuatro perfiles representativos en la conformación del movimiento en Facebook y Twitter. Los resultados apuntan a que ambas plataformas tienen efectos positivos en la movilización ciudadana, conformándose como espacios de disidencia, de articulación del movimiento y de organización de acciones-protesta.

Palabras clave: movimientos sociales, redes sociales, plataformas digitales, Facebook, Twitter.

\begin{abstract}
This research determines the role that social networks play in the organization of the social movement during the "Frijoleros Revolution" in Guatemala in 2020. Through a mixed approach that combines content analysis and critical discourse analysis, four representative profiles are analyzed in the conformation of the movement on Facebook and Twitter. The results suggest that both platforms have positive effects on citizen mobilization, forming spaces for dissent, for articulating the movement and organizing protest-actions.
\end{abstract}

Keywords: social movements, social media, digital platforms, Facebook, Twitter.

\section{Resumo}

Esta pesquisa determina o papel que as redes sociais desempenham na organização do movimento social durante a "Revolução Frijoleros" na Guatemala em 2020. Por meio de uma abordagem mista que combina análise de conteúdo e análise crítica do discurso, quatro perfis representativos são analisados na conformação do movimento no Facebook e no Twitter. Os resultados sugerem que ambas as plataformas têm efeitos positivos na mobilização cidadã, tornando-se espaços de dissidência, de articulação do movimento e de organização de ações de protesto.

Palavras-chave: movimentos sociais, redes sociais, plataformas digitais, Facebook, Twitter. 


\section{Introducción}

Internet y, en concreto, las redes sociales, han contribuido significativamente a transformar los modos de comunicación social. Según el informe anual Global Digital Overview (Hootsuite y We Are Social, 2021) más de la mitad de la población mundial ( $\mathrm{n}=3.800$ millones de personas, $53 \%$ ) utiliza actualmente las redes sociales; una cifra que se ha visto incrementada sustancialmente en este último año de pandemia. De los 10 países que lideran este ranking en cuanto a tiempo diario de uso, 4 son latinoamericanos: Colombia y Brasil (segundo y tercer puesto), México y Argentina (séptimo y octavo puesto). Poniendo el foco en el país donde se centra esta investigación, conforme al dossier Digital 2021 Local Country Headlines-Guatemala, el número de usuarios de redes sociales en Guatemala aumentó en 1,4 millones (+ 18\%) entre 2020 y 2021.

Gracias a esta intensificación en el uso y al alto grado de visibilidad que pueden proporcionar, los medios sociales se han convertido en instrumentos clave para la creación de espacios comunicativos de disidencia y organización de movimientos sociales.

Diversas investigaciones centradas en la participación cívica y política contemplan la utilización de las redes sociales en Iberoamérica (Matassi y Boczkowski, 2020). Muchos de estos análisis atestiguan, desde varias perspectivas, cómo se produce una predisposición en la utilización de los medios sociales como plataformas que coadyuvan en la organización, articulación de grupos de manifestantes y convocatoria de acciones de protesta (Cardoso y Di-Fátima, 2013; De-Melo-Romão, 2014). La creación de perfiles en el espacio digital secunda tanto a protestas moderadas como a radicales, pero se ha confirmado que los usuarios latinoamericanos utilizan en mayor medida las redes sociales para apoyar las primeras (Mourão, Saldaña, McGregor y Zeh, 2016).

Publicaciones de la última década muestran los efectos positivos de la utilización de las redes sociales, constatando que se produce un fuerte vínculo entre el uso de estas y la participación en las protestas (Boulianne, 2017; Tince, Liang y He, 2017; Gan, Lee y Li, 2017; Masías, Hecking y Hoppe, 2018; Kim y Chen, 2016; Salzman, 2015a; 2015b; Valenzuela, 2013).

Este nexo positivo entre la frecuencia del uso de las redes sociales y la participación en la acción política se ha examinado concretamente en los espacios de Facebook y Twitter en Chile, concluyéndose que ambas plataformas digitales proporcionan efectos positivos en la movilización de la ciudadanía y en el fomento de acciones propias de la protesta política, a través de mecanismos divergentes (Valenzuela, Arriagada y Scherman, 2012; Valenzuela, 2013; Valenzuela, Correa y Gil-de-Zúñiga, 2018).

Diversos trabajos sobre Guatemala (Harlow, 2012; Harlow y Harp, 2012) manifiestan que los medios sociales muestran mucho potencial y son de gran utilidad para el activismo, ayudando a organizar protestas masivas y centrando 
las demandas sociales, políticas y económicas (Harlow, 2012). Incluso revelan que redes sociales como Facebook y Twitter han contribuido a activar movimientos en modalidad online, traduciéndose posteriormente en un activismo offline en las calles (Harlow y Harp, 2012).

En Latinoamérica se tiene constancia de una importante experiencia en acciones colectivas que en la última década están empleando el medio digital como el instrumento primordial para la organización y atracción de simpatizantes. Como experiencias recientes se encuentran: \#YoSoy132 (México, 2012-2013), Ayotzinapa 43 (México, 2014), Movimiento estudiantil Chileno-La Primavera de Chile / Chile despertó (Chile, 2019-20/21), Movimiento estudiantil colombiano (2020) o las movilizaciones populares en Perú, Ecuador y Brasil (2020). Se trata de movilizaciones sociales que se han beneficiado de las oportunidades que ofrece el entorno digital y las redes sociales para cimentar procesos de participación ciudadana autónoma que han conseguido presionar para exigir cambios sociales y políticos, producto de aquellas demandas no satisfechas de la sociedad (Corrales-Mejías, 2015).

El antecedente en Guatemala, estrechamente relacionado con la temática del movimiento social surgido en 2020 , son las movilizaciones anticorrupción de 2015 organizadas a través de los medios sociales, que derivaron en el denominado movimiento \#RenunciaYa.

\subsection{La movilización social en Guatemala 2020}

Latinoamérica es una región con una amplia tradición en la movilización social, marcada por múltiples acciones-protesta y movimientos sociopolíticos de indignación en los últimos años que han desencadenado en varias ocasiones, en estallidos sociales.

Este es el caso de Guatemala que, como otras democracias de América Latina, ha sido testigo en 2020 de protestas y movilizaciones sociopolíticas. El surgimiento y desarrollo del movimiento social que se aborda en esta investigación, la "Revolución de los Frijoleros", se explica atendiendo a los siguientes antecedentes:

En la madrugada del 18 de noviembre de 2020, el Congreso guatemalteco, encabezado por su presidente Alejandro Giammattei que gobierna por mayoría, aprueba el mayor presupuesto de la historia del país para 2021. La decisión parlamentaria fue criticada por la ciudadanía y vetada por su vicepresidente de gobierno, Guillermo Castillo, que incluso instó a Giammattei a renunciar conjuntamente "por el bien del país". La reprobación del presupuesto se puede resumir en tres motivaciones: el fuerte endeudamiento para el país que supone la nueva propuesta económica, el alto porcentaje que conllevan los gastos destinados a personal parlamentario y la falta de inversión en educación, salud y la lucha contra la desnutrición; todo ello envuelto en una acción sorpresiva y acelerada que no ha permitido la discusión social. (Diálogo Político, 2020) 
Tras las comparecencias del presidente y vicepresidente del gobierno durante la jornada del 20 de noviembre, al día siguiente (21 de noviembre) tuvieron lugar las primeras protestas en el país encabezadas por las celebradas en la plaza principal de la ciudad de Guatemala, frente al Palacio de la Cultura y la Catedral (despacho del Gobierno), y secundadas por manifestaciones en las capitales que culminaron con violentos enfrentamientos de enmascarados que prendieron fuego al edificio del Parlamento (Elías, 2020).

Las peticiones principales de los manifestantes han sido: la renuncia del presidente Alejandro Giammattei y de los 115 diputados afines a su movimiento político, una convocatoria a una Asamblea Nacional Constituyente Popular y mayor transparencia en los procesos gubernamentales (La Vanguardia, 2020). Dichas demandas se han articulado a través de manifestaciones y protestas que han desembocado en la gestación del movimiento \#28N, que posteriormente se ha denominado la "Revolución de los Frijoleros" (Laguardia, 2020; GumucioRivas, 2020). Esta denominación responde a las declaraciones del diputado conservador del Partido Valor, Rubén Barrios, que se dirigió a los manifestantes despectivamente como "los comelones de frijoles" (Santos, 2020). El conflicto político-social ha requerido de la intervención de la Organización de Estados Americanos (OEA) como actor mediador, dictaminándose incluso medidas específicas sobre ciertos asuntos que preocupan a la sociedad comola corrupción o la impunidad política (Méndez, 2020). También han sido condenados por otros organismos internacionales, como Amnistía Internacional o la ONU, los incidentes que han enfrentado a manifestantes y fuerzas del orden público.

El movimiento social, al que paulatinamente han ido adhiriéndose diversos actores políticos y sociales, se ha ido gestando gracias a las redes sociales que han sido utilizadas como herramienta para la articulación y organización social valiéndose del empleo de hashtags en los distintos medios sociales. Prueba de ello es el empleado para el inicio del movimiento social, \#28N, que dio nombre originalmente a la movilización en sus comienzos. Posteriormente han ido viralizándose en muy poco tiempo otros que han servido como aglutinadores de las demandas sociales o como convocantes a las protestas: \#FrijolerosUnidos, \#RenunciaGiammattei, \#Presupuesto2021, SalimosTodos, \#GuatemalaSinMiedo, \#QueRenuncienTodos, \#AsambleaConstituyentePlurinacional, \#NoNosLaPela, \#VetoAlPresupuesto, \#PactoDeCorruptos o \#RenunciasYa.

Los niveles de penetración de los medios sociales en países latinoamericanos son actualmente bastante altos. Focalizando en el país que centra esta investigación y en las redes sociales que sirven de contexto al estudio, en Guatemala, a fecha de enero de 2021, las personas que utilizan Facebook ascienden a 8,5 millones, mientras que las que emplean Twitter son 515.600 (Global Digital Overview, 2021). Estos datos cobran relevancia para estudiar los efectos que estas plataformas pueden suponer en el comportamiento político de 
los usuarios, ya que dichos social media están omnipresentes en el día a día de la ciudadanía (Valenzuela et al., 2018).

\section{Objetivos y metodología}

Esta investigación persigue como principal objetivo, analizar el papel de las redes sociales en la organización del movimiento social y de las acciones colectivas durante la denominada "Revolución de los Frijoleros" en Guatemala en 2020.

De este objetivo general, se desprenden los siguientes objetivos específicos:

1. Examinar cuáles son las principales asociaciones, organismos o plataformas que han contribuido a la conformación de dicho movimiento social.

2. Delimitar el peso de cada una en la configuración de dicho movimiento social en la red, atendiendo al nivel de implicación en la convocatoria de protestas o concentraciones, así como al volumen de difusión de contenido vinculado al movimiento.

3. Comprobar cuáles son las redes sociales en las que tienen mayor actividad comunicativa con relación al movimiento social.

4. Determinarsisehaproducidouncambiodecomportamientosignificativo en sus perfiles online que indique si los contenidos publicados han tenido incidencia o no en la visibilidad del movimiento-protesta y la adhesión de usuarios afines a este.

5. Constatar hasta qué punto las redes sociales se constituyen como espacios comunicativos relevantes para la disidencia, articulación de movimientos sociales y organización de acciones protesta.

En el plano metodológico, desde una perspectiva exploratoria-descriptiva, se propone un enfoque mixto (cuantitativo-cualitativo) en el que se combina el análisis de contenido (Igartua, 2006; López-Aranguren, 2016) y el análisis crítico del discurso (Van Dijk,1999; 2016; Wodak, 2003); este último desde la óptica del análisis de la conversación (Tusón-Valls, 2002).

En la convocatoria del movimiento social analizado participan múltiples asociaciones, organismos o plataformas: Colectivo Estudiantil Universitario, Otra Guatemala Ya, Landivarianos, Movimiento Sindical Indígena y Campesino Guatemalteco, Unidos Contra la Corrupción, Jóvenes Políticos en Acción, Coincidir, Organización por los Derechos de Niñez y Adolescencia, Plataforma Cívica GT, Comité de Unidad Campesina, La Batucada del Pueblo, JusticiaYa, WaqibKej Convergencia, Movimiento Político WINAQ y el think tank ASIES.

De cara a seleccionar a los colectivos organizadores más representativos, se rastrearon los perfiles de todos ellos en las redes sociales más populares: Facebook, Twitter, Instagram y YouTube. En los perfiles explorados, se atiende a 
las cuentas que ofrecen mayor contenido propio relacionado con la temática de estudio - descartando, por tanto, aquellas otras que recogen un alto porcentaje de material compartido proveniente de otros perfiles-, y al hecho de que estas existieran con anterioridad a la gestación del movimiento; lo que posibilita examinar un posible cambio en el comportamiento de los datos registrados en la misma y vinculados a la aparición de la "Revolución de los Frijoleros".

Bajo estos parámetros, la muestra queda finalmente delimitada por dos perfiles representativos en Facebook: Colectivo Estudiantil Universitario (CEU) y el de Otra Guatemala Ya; y otros dos en Twitter: Landivarianos y Movimiento Sindical, Indígena y Campesino Guatemalteco (MSICG).

La selección de Facebook y Twitter como las redes sociales más significativas para este estudio frente a Instagram o YouTube viene determinada por ser estas las que registran, con relación al movimiento social que se examina, mayores índices en cuanto a frecuencia de publicación, número de seguidores e interacción.

Con base a estos cuatro perfiles, la muestra final atiende a 338 entradas/ posts y a 1765 comentarios, con distinto peso en función de la cuenta de la que proceden.

Tabla 1. Muestra final: entradas y comentarios

\begin{tabular}{|l|c|c|l|c|c|}
\hline $\begin{array}{c}\text { Comentarios } \\
\text { analizados }\end{array}$ & $\begin{array}{c}\text { N. En cada } \\
\text { perfil }\end{array}$ & $\begin{array}{c}\text { \% que representa } \\
\text { cada perfil }\end{array}$ & Posts analizados & $\begin{array}{c}\text { N. De cada } \\
\text { perfil }\end{array}$ & $\begin{array}{c}\text { \% que representa } \\
\text { cada perfil }\end{array}$ \\
\hline Ceu & 252 & $14,28 \%$ & Ceu & 44 & $13,01 \%$ \\
\hline Otra Guatemala ya & 914 & $51,78 \%$ & $\begin{array}{l}\text { Otra Guatemala } \\
\text { ya }\end{array}$ & 146 & $43,19 \%$ \\
\hline Landivarianos & 315 & $17,85 \%$ & Landivarianos & 83 & $24,56 \%$ \\
\hline Msicg & 284 & $16,09 \%$ & Msicg & 65 & $19,24 \%$ \\
\hline Total & 1765 & $100 \%$ & Total & 338 & $100 \%$ \\
\hline
\end{tabular}

Fuente: elaboración propia

La acotación temporal del análisis se circunscribe a dos meses. En concreto al periodo comprendido entre el 18 de octubre y el 19 de diciembre de 2020. Esta selección viene motivada por el momento en el que surge el movimiento y se inician las acciones de protesta. El 18 de noviembre de 2020, de madrugada, el Congreso de Guatemala aprueba el mayor presupuesto de la historia del país, respaldado por su presidente, Alejandro Giammattei. Tras este hecho, comienzan a tener lugar concentraciones y marchas que se suceden entre finales del mes de noviembre y la primera mitad del mes de diciembre. Se considera también en el estudio el periodo inmediatamente anterior (del 18 de octubre al 18 de noviembre) con el objeto de determinar, a nivel cuantitativo, si se produce 
un cambio de comportamiento determinante en los perfiles seleccionados que pueda constatar el papel que cada una de las organizaciones consideradas han jugado en relación a la visibilidad y la adhesión al movimiento contestatario.

En el diseño de la plantilla de análisis se han tenido en cuenta diversas variables y categorías cuya pertinencia se fundamenta en el uso que, de las mismas, se constata en estudios precedentes (Farné e Iranzo, 2018; Gil-Ramírez, Gómez-de-Travesedo-Rojas \& AlmansaMartínez, 2020; Smolak, López, Gómez \& Castillo, 2018).

Tabla 2. Plantilla de análisis

\begin{tabular}{|c|c|}
\hline \multicolumn{2}{|l|}{ Datos identificativos } \\
\hline \multicolumn{2}{|l|}{$\begin{array}{l}\text { Red Social: Facebook / Twitter } \\
\text { Nombre del perfil: }\end{array}$} \\
\hline \multicolumn{2}{|l|}{ Análisis formal-descriptivo } \\
\hline Variables & Categorías \\
\hline Número de seguidores & $\begin{array}{l}\text { N. de seguidores totales } \\
\text { N. de seguidores por mes }\end{array}$ \\
\hline Número de posts/entradas & $\begin{array}{l}\text { N. de posts/entradas totales } \\
\text { N. de posts/entradas por mes (frecuencia de publicación) }\end{array}$ \\
\hline \multicolumn{2}{|l|}{ Análisis de contenido publicado } \\
\hline Variables & Categorías \\
\hline Post/entrada relacionada con el movimiento social & $\begin{array}{l}\text { Sí } \\
\text { No }\end{array}$ \\
\hline Tipo de post/entrada & $\begin{array}{l}\text { Informativo } \\
\text { Reivindicativo } \\
\text { Infoentretenimiento }\end{array}$ \\
\hline Lenguaje empleado en el post/entrada (texto) & $\begin{array}{l}\text { Agresivo } \\
\text { Crítico/Reivindicativo } \\
\text { Satírico/Irónico } \\
\text { Neutro/Indeterminado }\end{array}$ \\
\hline Tipo de fotografía/imagen (temáticas) & $\begin{array}{l}\text { Informativa/ilustrativa } \\
\text { Reivindicativa } \\
\text { Satírica }\end{array}$ \\
\hline Tipo de vídeo (temáticas) & $\begin{array}{l}\text { Informativo } \\
\text { Reivindicativo } \\
\text { Infoentretenimiento }\end{array}$ \\
\hline \multicolumn{2}{|l|}{ Análisis de la interacción/participación } \\
\hline Variables & Categorías \\
\hline Nivel de interacción/participación & $\begin{array}{l}\text { N. de reacciones por mes } \\
\text { N. de comentarios por mes } \\
\text { Contenido compartido por mes }\end{array}$ \\
\hline Tipo de reacción en la conversación del post & $\begin{array}{l}\text { Positiva } \\
\text { Negativa } \\
\text { Neutra }\end{array}$ \\
\hline $\begin{array}{l}\text { Tipo de comentario en función del nivel de razonamiento } \\
\text { en la exposición del mismo }\end{array}$ & $\begin{array}{l}\text { Con carga argumentativa/reflexiva } \\
\text { Con carga emocional/visceral } \\
\text { Vacío }\end{array}$ \\
\hline
\end{tabular}

Farné e Iranzo (2018); Gil-Ramírez et al.(2020); Smolak et al. (2018) 
Las categorías del análisis formal-descriptivo ayudan a determinar si los perfiles analizados han contribuido a dar mayor visibilidad al movimiento de protesta guatemalteco a través de los posts/entradas publicados, así como a sumar adeptos al mismo.

Las categorías referentes al análisis del contenido publicado por cada perfil permiten una aproximación a la tipología del contenido que se publica en estos espacios; lo que nos brinda una idea de la implicación de estos perfiles en la conformación de movimientos sociales. El hecho de que el contenido publicado en el periodo analizado esté centrado casi en su totalidad en el movimiento social, dejando de lado otras temáticas, confirmaría la idea de que las redes sociales constituyen una plataforma consistente para articular movimientos sociales, ya que suponen espacios que coadyuvan tanto a difundir información como a documentar y mostrar contenido multimedia que testimonie las actividades de la movilización social, ya sea dentro o fuera de la red (Baym, 2015; Kruikemeier y Shehata, 2016; Masías, Hecking y Hoppe, 2018; Skoric et al., 2011). Generando, además, impacto emocional a través de los documentos fotográficos y audiovisuales, que se convierten en instrumentos motivacionales para los usuarios (Cmeciu y Coman, 2016).

Por otra parte, las variables cuantitativas que se contemplan en este bloque permiten indagar sobre la forma particular de comunicar que tiene cada perfil para atraer usuarios afines al movimiento.

Para la inclusión de las categorías que componen estos dos primeros bloques se toman como referencia las contribuciones de García de Torres et al. (2011), Holt (2004), Sampedro (2011), Farné e Iranzo (2018) y "Smolak et al" (2018).

Con relación a la última variable que analiza la interacción/participación de los usuarios que intervienen en los perfiles, la presente investigación se apoya en el trabajo de Smolak et al (2018) para determinar el tipo de reacción que se genera en la conversación del post. Solo se han tenido en cuenta los comentarios a las entradas relacionadas con el movimiento, para precisar si apoyan o se adhieren a este, mostrándose a favor de las actividades y opiniones vinculadas a las protestas (reacción positiva), si se muestran en contra (reacción negativa) o si simplemente el comentario resulta neutral.

Este mismo criterio se ha aplicado en la categoría referida a examinar el tipo de comentario en función del nivel de razonamiento en la exposición del mismo, atendiendo únicamente a las entradas relacionadas con la temática de las acciones-protesta y el movimiento social. La incorporación al presente estudio de las variables de comunicación que se contemplan en este bloque se fundamenta en la propuesta de Gil-Ramírez et al. (2020).

Esta variable contribuye a calibrar el apoyo real al movimiento en redes sociales a través del tipo de reacciones según los comentarios a los posts publicados. Igualmente, el examen del tipo de comentario en función del nivel de razonamiento en la exposición del mismo, permite determinar el grado de implicación de los seguidores y simpatizantes. 
Durante el curso de la investigación se han desechado del análisis aquellas entradas que son contenido compartido de otra cuenta, si no se ha hecho aportaciones nuevas - a nivel textual - desde el perfil analizado, dado que no se consideraría material propio de este. Además, en el examen de los comentarios por parte de los usuarios, se han descartado aquellos que solo están conformados por fotografías, ilustraciones, enlaces a vídeos o etiquetas y menciones a perfiles de personas. Tampoco se han tenido en cuenta los comentarios agrupados o grupo de conversaciones, es decir, las contestaciones a comentarios de usuarios. En el análisis de la conversación se contemplan únicamente aquellos comentarios individuales que los usuarios han hecho en referencia al contenido publicado por la cuenta analizada. De este modo, el total de 1765 comentarios que conforman la muestra, provienen de los 10 primeros comentarios entrantes en cada post examinado (Gil-Ramírez et al., 2020).

Para el análisis de las variables cuantitativas se emplearon las herramientas Metricool y SocialGest, que permiten examinar las métricas en redes sociales, suministrando datos vinculados a seguidores, frecuencia de publicación y nivel de interacciones.

De cara a validar el diseño de la plantilla de análisis, se realizó un estudio exploratorio preliminar de dos de los cuatro perfiles escogidos para la muestra, uno en Facebook y otro en Twitter. Esta primera evaluación permitió considerar la idoneidad de la metodología, modificando y reajustando posibles elementos que dificultarán alcanzar los objetivos propuestos. Finalmente, la plantilla de análisis fue aplicada por dos codificadores (EC y MG) a la totalidad de la muestra. Se empleó el coeficiente kappa de Cohen para evaluar el nivel de concordancia, resultando $\mathrm{k}=0,88$.

\section{Resultados}

\subsection{De naturaleza formal-descriptiva}

Con relación a los datos referentes a los seguidores de los distintos perfiles, atendiendo a una comparativa entre los meses estudiados, se evidencia que el número ha crecido considerablemente en el período en el que se originan las distintas acciones colectivas (19 de noviembre-19 de diciembre de 2020) respecto al inmediatamente anterior (18 de octubre-18 de noviembre de 2020). Destacan el perfil de CEU que multiplica por más de 6 los seguidores de la página. Tanto en el caso de Landivarianos como en el de MSICG, los seguidores se cuadriplican a raíz del surgimiento de la protesta; mientras que en la cuenta de Otra Guatemala Ya, la cifra se triplica. Estos incrementos evidencian un creciente interés ciudadano hacia los argumentos que defienden las organizaciones del movimiento social objeto de estudio ("Revolución de los Frijoleros"). 
Tabla 3. Número de seguidores de los perfiles analizados

\begin{tabular}{|l|l|c|c|c|}
\hline & \multicolumn{1}{|c|}{$\begin{array}{c}\text { N. De seguidores } \\
\text { totales* }\end{array}$} & $\begin{array}{c}\text { Nuevos seguidores } \\
\text { 18 Oct.-18 Nov. 2020 }\end{array}$ & $\begin{array}{c}\text { Nuevos seguidores } \\
\text { 19 Nov.-19 Dic. 2020 }\end{array}$ \\
\hline \multirow{3}{*}{ Facebook } & CEU & 38.550 & 47 & 284 \\
\cline { 2 - 5 } & Otra Guatemala Ya & 50.689 & 61 & 186 \\
\hline \multirow{3}{*}{ Twitter } & Landivarianos & 6.223 & 33 & 136 \\
\cline { 2 - 5 } & MSICG & 2.278 & 21 & 62 \\
\hline \multirow{2}{*}{ *N. de seguidores totales a fecha del 19 de diciembre de 2020. } \\
\hline
\end{tabular}

Fuente: elaboración propia a partir de los datos extraídos de Metricool y SocialGest

Comprobando la cantidad de entradas publicadas por cada perfil, teniendo en cuenta los dos meses examinados, a excepción de la cuenta de Otra Guatemala Ya, el resto publica más posts/entradas tras el surgimiento del movimiento social —entre el 19 noviembre y el 19 diciembre—, resaltando los perfiles en Twitter de MSICG, que quintuplica el número de tuits emitidos y la de Landivarianos, que multiplica las entradas por más del doble. En este caso, los incrementos constatan, en términos generales, la implicación por parte de las organizaciones con el movimiento a través de sus redes sociales y un uso comunicativo estratégico de las mismas.

Tabla 4. Número de posts/entradas de los perfiles analizados

\begin{tabular}{|c|c|c|c|c|}
\hline & & $\begin{array}{l}\text { N. De posts/entradas totales } \\
\text { del perfil }{ }^{*}\end{array}$ & $\begin{array}{l}\text { Nuevas entradas } \\
18 \text { Oct.-18 Nov. } 2020\end{array}$ & $\begin{array}{l}\text { Nuevas entradas } \\
19 \text { Nov.-19 Dic. } 2020\end{array}$ \\
\hline \multirow{2}{*}{ Facebook } & CEU & $347^{\star \star}$ & 31 & 44 \\
\hline & Otra Guatemala Ya & $1.514^{\star \star}$ & 176 & 146 \\
\hline \multirow{2}{*}{ Twitter } & Landivarianos & 2.566 & 36 & 83 \\
\hline & MSICG & 1.483 & 13 & 65 \\
\hline
\end{tabular}

Fuente: elaboración propia a partir de los datos extraídos de Metricool y SocialGest.

La particularidad del caso de Otra Guatemala Ya viene dada porque, en este estudio, se excluyen las entradas que son contenido compartido de otra cuenta y en las que no se han hecho aportaciones nuevas a nivel textual; comportamiento propio de este perfil, que suele recurrir a esta práctica y en el que, si tomamos la totalidad de las entradas sin exclusiones, estas suman en los dos períodos de estudio: 181 y 202 respectivamente. 


\subsection{Contenido publicado}

Entre los 338 posts/entradas examinados durante el periodo comprendido entre el 19 de noviembre y el 19 de diciembre de 2020, prácticamente la totalidad, el $92,6 \%$ ( $n=313)$, se corresponde con contenido relacionado con la gestación, formación y organización del movimiento social o con alguna de sus principales demandas. El resto $(7,4 \%, n=25)$ versa sobre otro tipo de temáticas, como información sobre hechos circunstanciales acaecidos en esas fechas o conmemoraciones de efemérides (véase el 25 de noviembre, Día Internacional de la Eliminación de la Violencia contra la Mujer). Entre los perfiles que suben posts sin vinculación alguna con la "Revolución de los Frijoleros", destaca el espacio en Facebook de Otra Guatemala Ya, en el que un 14,38\% (n=21) de los contenidos tratan asuntos distintos al movimiento social objeto de estudio. En el extremo opuesto se sitúa MSICG, en cuya cuenta de Twitter solo se atiende al movimiento contestatario.

Gráfico 1. Entradas vinculadas al movimiento social y otras temáticas

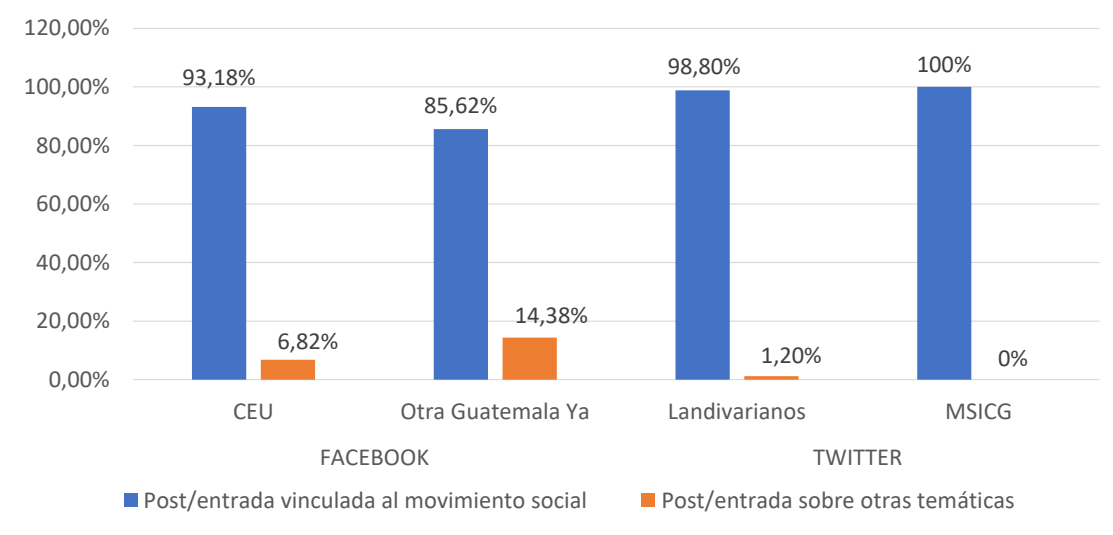

Fuente: elaboración propia

Tras constatar el peso que suponen los posts vinculados con el movimiento social en el periodo analizado con relación a otra tipología de contenido que se venía publicando en el perfil, las siguientes variables empleadas para el análisis del contenido publicado en los espacios de las cuatro organizaciones seleccionadas se focalizan sobre aquellos contenidos que tratan específicamente sobre la protesta guatemalteca $(n=313)$, dado que aquellos posts/entradas que abordan temáticas distintas $(n=25)$ carecen de interés para la consecución del resto de objetivos propuestos. 


\subsubsection{Tipo de post/entrada}

En cuanto a la tipología de los contenidos publicados desde los perfiles examinados, prevalece el carácter reivindicativo (67,73\%, n=212). En los espacios analizados, tanto en Facebook como en Twitter, se supera el 60\% en los posts que giran en torno a algún tipo de reivindicación de entre las que postula el movimiento contestatario. Resulta especialmente destacado el perfil de MSICG en Twitter, donde el porcentaje de publicaciones de esta naturaleza asciende al 75,38\% ( $\mathrm{n}=49)$. La función informativa que realizan las principales organizaciones que gestaron la protesta entre los usuarios de sus perfiles también queda patente en los contenidos que publican, siendo la información el eje central en un $30,99 \%(n=97)$ de los contenidos. El recurso al sensacionalismo o al humor, sin embargo, no es algo que caracterice la comunicación de los organizadores del movimiento social guatemalteco a través de sus redes sociales, quedando el uso del infoentretenimiento relegado únicamente al perfil en Facebook de Otra Guatemala Ya, que, en cualquier caso, lo emplea de forma anecdótica $(1,28 \%, \mathrm{n}=4)$.

Gráfico 2. Tipo de post/entrada

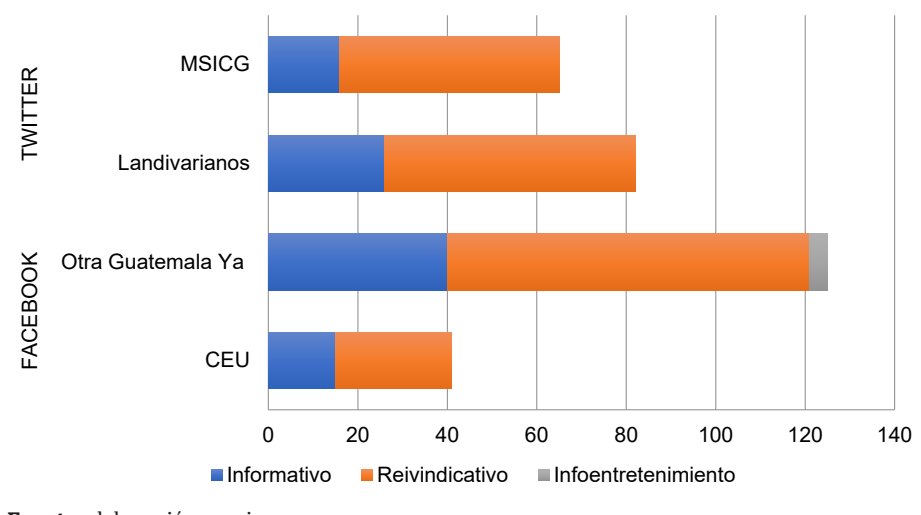

Fuente: elaboración propia

\subsubsection{Lenguaje empleado en el post/entrada (texto)}

Acerca del lenguaje que las organizaciones del movimiento social emplean en los posts/entradas que publican en sus perfiles predomina la jerga crítica o reivindicativa $(57,14 \%, \mathrm{n}=156)$. Este porcentaje resulta cercano a los resultados obtenidos en cuanto a la tipología de los posts/entradas, donde también prima la reivindicación. 
Gráfico 3. Lenguaje empleado en el post/entrada (texto)

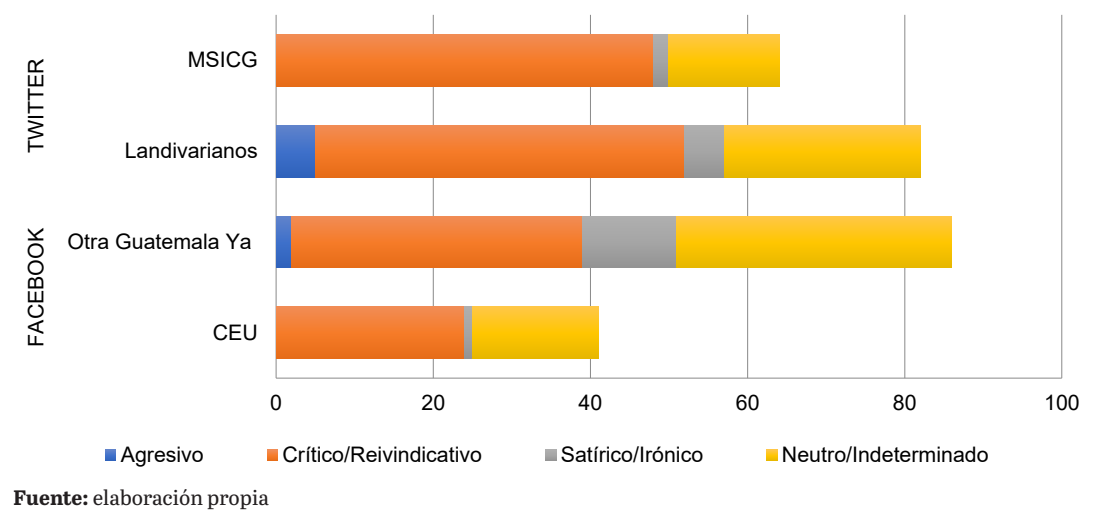

Al margen de la preponderancia de la terminología vinculada a exigencias, demandas, protestas, críticas o reivindicaciones en el tipo de comunicación que las organizaciones del movimiento contestatario emplean en sus perfiles, tan solo resulta reseñable, en el resto de los contenidos publicados, el uso de un lenguaje en un tono neutro (32,97\%, $\mathrm{n}=90)$. En términos generales, no es habitual por parte de las organizaciones de la "Revolución de los Frijoleros" utilizar en sus publicaciones expresiones satíricas o irónicas $(7,33 \%, \mathrm{n}=20)$, siendo su uso demostrativo principalmente en el perfil de Otra Guatemala Ya $(13,95 \%, n=12)$. La agresividad verbal resulta un aspecto residual $(2,56 \%, n=7)$ en el modo en que las organizaciones contempladas se comunican con los usuarios de sus perfiles en redes sociales. Este tipo de lenguaje impositivo, violento, incívico o dañino solo está presente en dos de las cuentas examinadas (Otra Guatemala Ya y Landivarianos) y, en cualquier caso, en porcentajes poco significativos.

\subsubsection{Empleo de fotografía/imagen o vídeo y sus temáticas}

El empleo de elementos ilustrativos que acompañen o complementen al texto en los posts/entradas publicados por las organizaciones del movimiento social guatemalteco es mayoritario $(88,18 \%, \mathrm{n}=276)$; si bien, atendiendo a la comparativa entre los perfiles analizados, el espacio de MSICG en Twitter destaca por su tendencia a la baja a la hora de utilizar elementos ilustrativos junto al texto, solo el $61,76 \%$ de sus entradas los utilizan ( $n=42)$; mientras que CEU resulta la organización que más recurre a esta práctica en su página de Facebook, ya que tan solo el 2,44 \% ( $n=1)$ de sus publicaciones son exclusivamente textuales. En términos generales, las imágenes o fotografías son un recurso más recurrente que los vídeos $(67,03 \%, \mathrm{n}=185$ frente a $32,97 \%, \mathrm{n}=91)$. Sin embargo, el uso que se hace de estos elementos difiere en función de la red social: en Facebook (CEU y Otra Guatemala Ya), fotografías y vídeos se utilizan de forma más o menos 
equilibrada (55,28\%, n=89 y 44,72\%, $\mathrm{n}=72$, respectivamente), mientras que en Twitter (MSICG y Landivarianos) se recurre con mucha más asiduidad al empleo de fotografías $(83,48 \%, \mathrm{n}=96)$ que de vídeos $(16,52 \%, \mathrm{n}=19)$.

Por otra parte, atendiendo a la tipología de las ilustraciones, se evidencia cierta concordancia con los resultados obtenidos, tanto con relación al contenido, como al lenguaje empleado en los posts/entradas. También en el uso que desde los perfiles se realiza de los elementos que complementan al texto priman, de forma más o menos equilibrada, los gráficos y vídeos de índole informativa y los de carácter reivindicativo ( $50 \%, \mathrm{n}=138$ y 44,57\%, $\mathrm{n}=123$, respectivamente). Si bien, la información es más representativa a través de la utilización que se hace de las imágenes/fotografías (40,94\%, n=113), mientras que la reivindicación impregna, de forma repartida, tanto los elementos exclusivamente visuales, como los audiovisuales (21,38\%, $\mathrm{n}=59$ y $23,19 \%, \mathrm{n}=64$, respectivamente).

Gráfico 4. Empleo de fotografía/imagen o vídeos ilustrativos y sus temáticas

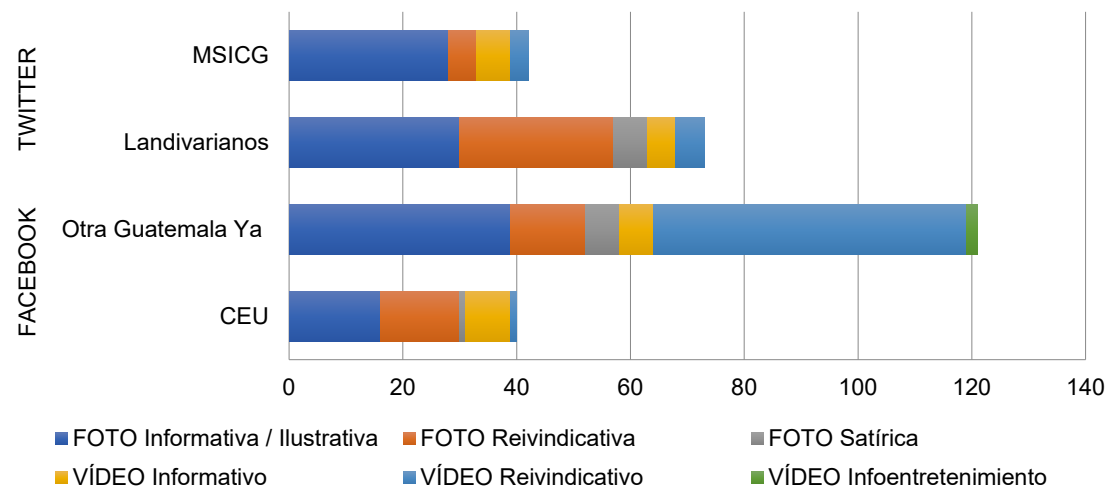

Fuente: elaboración propia

Al focalizar en el tipo de ilustración que emplean cada uno de los perfiles examinados, la comparativa constata diferentes tendencias entre las organizaciones que se contemplan en este estudio. En las fotografías y vídeos que MSICG (Twitter) y CEU (Facebook) emplean para ilustrar sus posts/entradas predomina el tono informativo (80,96\%, $\mathrm{n}=34$ y el $60 \% \mathrm{n}=24$, respectivamente), Otra Guatemala Ya se caracteriza por el marcado estilo reivindicativo de las ilustraciones en su página de Facebook $(56,19 \%, n=68)$, y Landivarianos (Twitter) resulta el perfil más equilibrado en este sentido, utilizando casi en la misma medida elementos informativos y reivindicativos en las fotografías y vídeos con los que acompaña el texto en sus posts $(47,95 \%, \mathrm{n}=35$ y $43,84 \%, \mathrm{n}=32$, respectivamente).

De nuevo el infoentretenimiento o la sátira tienen una escasa presencia. El primero solo se manifiesta como complemento del texto en un $1,66 \%(n=2)$ de los vídeos publicados en el perfil de Otra Guatemala Ya; mientras que la fotografía 
satírica, pese a emplearse en tres de los cuatro perfiles investigados, arroja porcentajes insignificantes en el conjunto.

\subsection{Análisis de la interacción/participación}

\subsubsection{Interacciones y contenido compartido}

Del análisis del nivel de la interacción que los usuarios han mostrado en relación a los perfiles investigados se desprende que, en las tres categorías examinadas —número de reacciones, número de comentarios y contenido compartido-, la participación es notablemente mayor en el periodo en el que tiene lugar la gestación del movimiento social —entre el 19 de noviembre y el 19 de diciembre (en adelante "segundo periodo") — que en el período anterior — del 18 de octubre al 18 de noviembre- (en adelante "primer periodo"). Resulta prominente la cuenta de Otra Guatemala Ya como perfil que aglutina mayores interacciones en el segundo periodo evaluado en todas las categorías, a pesar de haber publicado más entradas en el primero. En términos porcentuales, el perfil más destacado es el de CEU, que multiplica en el segundo periodo por más de 14 veces las interacciones registradas durante el primero; casi 8 veces el número de comentarios generados en las publicaciones y más de 26 el contenido compartido de la cuenta. En líneas generales, todos los perfiles examinados experimentan incrementos en la interacción de sus usuarios en las tres categorías propuestas entre el segundo y el primer periodo de análisis, lo que evidencia un aumento en el nivel de compromiso de los seguidores vinculado al movimiento social la "Revolución de los Frijoleros".

Tabla 5. Nivel de interacción-participación en los perfiles analizados

\begin{tabular}{|l|l|l|l|l|l|l|l|}
\hline & & \multicolumn{2}{|c|}{ N. DE REACCIONES } & \multicolumn{2}{|c|}{$\begin{array}{r}\text { N. DE } \\
\text { COMENTARIOS }\end{array}$} & \multicolumn{2}{c|}{$\begin{array}{c}\text { CONTENIDO } \\
\text { COMPARTIDO }\end{array}$} \\
\hline \multirow{3}{*}{ FACEBOOK } & & $\begin{array}{l}18 \text { oct. } \\
18 \text { nov. } \\
2020\end{array}$ & $\begin{array}{l}19 \text { nov. } \\
19 \text { dic. } \\
2020\end{array}$ & $\begin{array}{l}18 \text { oct. } \\
18 \text { nov. } \\
2020\end{array}$ & $\begin{array}{l}19 \text { nov. } \\
19 \text { dic. } \\
2020\end{array}$ & $\begin{array}{l}18 \text { oct. } \\
18 \text { nov. } \\
2020\end{array}$ & $\begin{array}{l}19 \text { nov. } \\
19 \text { dic. } \\
2020\end{array}$ \\
\cline { 3 - 9 } & & 2.544 & 37.264 & 244 & 1.932 & 883 & 22.995 \\
\hline \multirow{3}{*}{ TWITTER } & Otra Guatemala Ya & 30.314 & 56.751 & 3.474 & 8.091 & 21.101 & 22.263 \\
\cline { 2 - 9 } & Landivarianos & 5.106 & 26.259 & 67 & 437 & 2.098 & 6.622 \\
\cline { 2 - 9 } & MSICG & 2.955 & 15.607 & 132 & 355 & 2.745 & 14.792 \\
\hline
\end{tabular}

Fuente: elaboración propia a partir de los datos extraídos de Metricool y SocialGest

\subsubsection{Tipo de reacción en la conversación del post}

Las reacciones de los usuarios de los cuatro perfiles observados, manifestadas a través de los comentarios que estos realizan a los posts/entradas que publican 
las organizaciones, permiten constatar un amplio apoyo al movimiento social contestatario, ya que 8 de cada 10 opiniones resultan positivas $(80,91 \%$, $\mathrm{n}=1428)$. De entre las que no lo son, la mayoría pueden ser consideradas como neutrales $(14,22 \%, \mathrm{n}=251)$ al no manifestarse de forma clara ni a favor, ni en contra de los postulados que esgrimen los organizadores de la "Revolución de los Frijoleros". Entre los perfiles examinados destaca MSICG (Twitter), en el que no se ha detectado atisbo alguno de reacciones negativas y en el que se presenta un porcentaje prácticamente unitario de comentarios que secundan las acciones del movimiento social $(97,54 \%, \mathrm{n}=277)$. Las opiniones que se oponen o contrarían los posts/entradas publicadas desde las plataformas gestoras son muy minoritarias $(4,87 \%, \mathrm{n}=86)$.

Gráfico 5. Tipo de reacción en la conversación del post

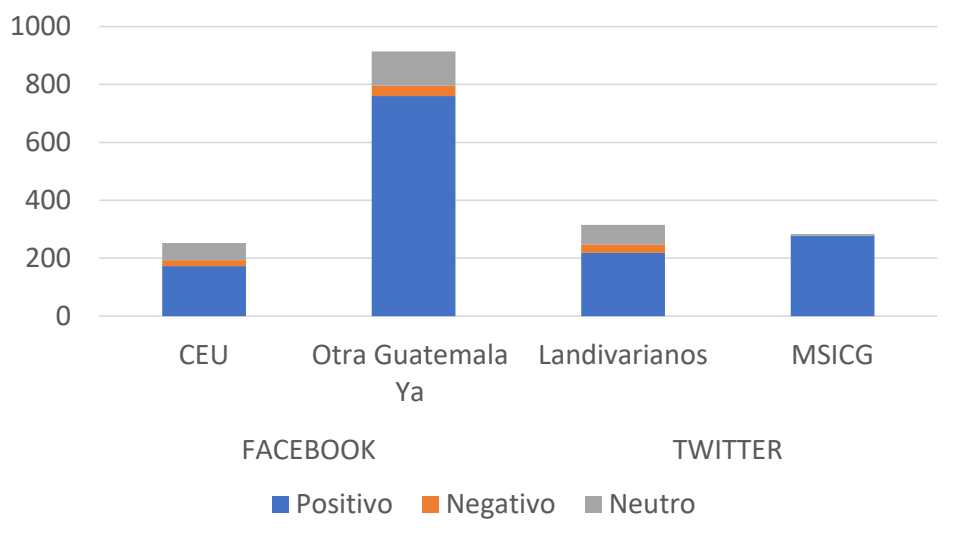

Fuente: elaboración propia

\subsubsection{Tipo de comentario en función del nivel de razonamiento en su exposición}

Con relación al nivel de razonamiento que los usuarios emplean en la exposición de sus comentarios, predominan los argumentos construidos desde posiciones subjetivas y con gran carga emocional $(75,94 \%, \mathrm{n}=1358)$. El perfil de MSICG en Twitter es el que aglutina un mayor número de comentarios marcados por el componente emocional-visceral $(93,31 \%, n=265)$, copando prácticamente la totalidad de la participación textual de los usuarios. Resultan poco representativas respecto al conjunto, tanto aquellas opiniones que utilizan términos vacíos y sin vinculación aparente con el tema tratado en el contenido publicado por el perfil $(15,41 \%, n=272)$, como aquellas que plantean una reflexión o argumentan con hechos contrastables o fuentes autorizadas $(8,65 \%, \mathrm{n}=135)$. 
Gráfico 6. Tipo de comentario en función del nivel de razonamiento en su exposición

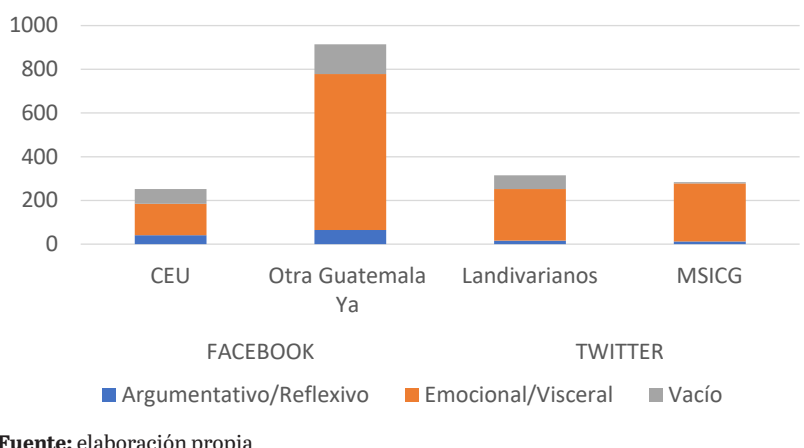

Fuente: elaboración propia

\section{Discusión y conclusiones}

En esta investigación, coincidiendo con estudios previos (Van-Laer y Van-Aelst, 2010; Sánchez-Duarte y Fernández-Romero, 2017), se ha constatado el poder de los medios digitales para la movilización y sensibilización de la ciudadanía sobre acontecimientos políticos de indudable incidencia política y social. Del análisis de los perfiles en las redes sociales revisadas se infiere una capacidad movilizadora intensa, reflejada en el incremento significativo de seguidores de los perfiles estudiados con respecto a periodos anteriores que indican la afinidad con los postulados publicados por las organizaciones que conforman el movimiento. Asimismo, se evidencia el uso de espacios comunicativos digitales con el objeto de dar visibilidad al movimiento contestatario la "Revolución de los Frijoleros", ya que las cuentas de estos organismos han vinculado casi la totalidad del contenido con las demandas, convocatorias de manifestaciones y otras acciones colectivas de este; desligándose estas temporalmente de otras temáticas sobre las que venían difundiendo información y contribuyendo de esta manera a la gestación de este movimiento. Refrendado por estudios previos (Ayala, 2014; Cabalín-Quijada, 2014; García-Galera, Del-Hoyo-Hurtado y Fernández-Muñoz, 2014; Giraldo-Luque, Fernández-García y Pérez-Arce, 2018; Valenzuela, 2013), ha quedado patente que los medios sociales están siendo el instrumento principal para la convocatoria de las acciones colectivas, consiguiendo una mayor visibilidad, participación y movilización social.

Sobre el uso que se hace de Facebook y Twitter para movilizar a la ciudadanía impera una función reivindicativa sobre los acontecimientos, seguida de una función informativa y aparece, de manera marginal, el infoentretenimiento. Este resultado muestra claramente el papel de movilización social como elemento de activismo ciudadano en tiempo real (Harlow, 2012; Ziccardi, 1998; Boulianne, 2017). Consecuencia de esa preponderancia del carácter reivindicativo, el lenguaje empleado utiliza expresiones críticas y de exigencia hacia la clase política. 
Sobre el empleo de recursos audiovisuales, en los perfiles analizados imperan las imágenes y las fotografías frente al vídeo en el caso de Twitter. Por el contrario, en Facebook existe un equilibrio en la utilización de ambos elementos. Esa inclusión de formas expresivas audiovisuales supone la constatación de que los espacios virtuales contienen procesos interactivos con contenido en línea (Suau-Gomila, 2015; Corrales-Mejías, 2015), que no solo posibilitan la organización social, sino la generación de contenido mediante el lenguaje audiovisual que permite relatar los conflictos sociales (Avalos, 2019).

En virtud de los resultados, se constata que en dichas plataformas se recogen principalmente apoyos y opiniones que secundan el movimiento social y se aprecia que el grado de implicación de los participantes en estos medios sociales muestra una significativa carga emocional con permanentes apelaciones a la movilización y al activismo social (Espino-Sánchez, 2014; Cmeciu y Coman, 2016). Dicho comportamiento demuestra la trascendencia de estos espacios digitales como posibilitadores para la discusión de ideas que pueden apoyar a la acción de ciudadanos que ya eran activistas en el entorno offline (Shirky, 2011), así como intensificadores de las probabilidades de participación del resto de la ciudadanía (Espino-Sánchez, 2014). Constituyéndose estos como un espacio público accesible que fomenta el diálogo, la interacción (Treré y Cargnelutti, 2014), así como el activismo cívico digital que muestra las exigencias dirigidas a la esfera social, económica, política, educativa o cultural (Cmeciu y Coman, 2016).

Finalmente, consideramos que la presente investigación podría suponer un punto de partida para futuros estudios acerca de otros fenómenos de la movilización social a través del medio digital en países de América Latina; extendiendo el análisis también a otras plataformas sociales que permitan corroborar los resultados obtenidos hasta el momento.

\section{Referencias bibliográficas}

Avalos González, J. M. (2019). La posibilidad tecnopolítica. Activismos contemporáneos y dispositivos para la acción. Los casos de las redes feministas y Rexiste. Comunicación $y$ Sociedad, 16, 1-30. https://doi.org/10.32870/cys.v2019io.7299

Ayala P., T. (2014). Redes sociales, poder y participación ciudadana. Revista Austral de Ciencias Sociales, 26, 23-48. https://www.redalyc.org/articulo.oa? id=459/45931862002

Baym, N. K. (2015). Personal connections in the digital age. Digital media and society series. PolityPress. https://bit.ly/3wLCgrH

Berrocal-Gonzalo, S., Redondo-García, M. y Campos-Domínguez, E. (2012). Una aproximación al estudio del infoentretenimiento en Internet: origen, desarrollo y perspectivas futuras. AdComunica Revista de Estrategias, Tendencias e Innovación en Comunicación, 4, 63-79. https://doi.org/10.6035/2174-0992.2012.4.5

Boulianne, S. (2017). Revolution in themaking? Social media effectsacrosstheglobe. Information, Communication \& Society, 22(1), 1-16. https://doi.org/10.1080/1369118X.2017.1353641

Cabalín-Quijada, C. (2014). Estudiantes conectados y movilizados: El uso de Facebook en las protestas estudiantiles en Chile. Comunicar, 22(43), 25-33. https://doi.org/10.3916/ C43-2014-02 
Cardoso, G. \& Di-Fátima, B. (2013). Movimentoem rede e protestos no Brasil: qual gigante acordou? Revista ECO-Pós, 16(2), 143-176. https://doi.org/10.29146/eco-pos.v16i2.1182

Cmeciu, C. y Coman, C. (2016). Activismo cívico digital en Rumanía: La comunidad de Facebook en las protestas on-line contra Chevron. Comunicar, 24, 19-28. https://doi. org/10.3916/C47-2016-02

Corrales-Mejías, R. (2015). Impacto de las redes sociales sobre la participación ciudadana en procesos electorales y la democracia: caso de Costa Rica. Consejo Latinoamericano de Ciencias Sociales (Clacso).http://biblioteca.clacso.edu.ar/clacso/becas/20150708062655/ Impacto Redes Sociales.pdf

De-Melo-Romão, W. (2014). \#naovaitercopa: manifestações, Copa do Mundo e as eleições de 2014. Agenda política, 1(2), 152-167.http://www.agendapolitica.ufscar.br/index.php/ agendapolitica/article/view/22/19

Diálogo Político (2020). Guatemala: el presupuesto de la discordia. Diálogo Político, 26 noviembre.https://dialogopolitico.org/agenda/guatemala-el-presupuesto-de-la-discordia/

Elías, J. (21 de noviembre de 2020). Cientos de manifestantes toman el Congreso de Guatemala y le prenden fuego. El País. https://elpais.com/internacional/america-latina/2020-11-21/cientos-de-manifestantes-toman-el-congreso-de-guatemala-y-le-prenden-fuego.html

Espino-Sánchez, G. (2014). La política en Internet, ¿de la mediatización a la convergencia digital? Convergencia. Revista de ciencias sociales, 65, 39-63.http://www.scielo.org.mx/ scielo.php?script $=$ sci_arttext\&pid $=\mathrm{S} 14.05-14352014000200002$

Farné, A. e Iranzo, A. (2018). Modelo de análisis cuantitativo-cualitativo de la comunicación para el cambio social en redes sociales: posibilidades y limitaciones metodológicas. En R. Eguizábal-Maza (Ed.), Metodologías 4. (p. 143-153) Fragua. http://hdl.handle. net/10234/180868

Gan, Ch.; Lee, F. L. F. y Li, Y. (2017). Social media use, political affect and participation among university students in urban China. Telematics and Informatics, 34(7), 936-947.https:// doi.org/10.1016/j.tele.2017.04.002

García-De-Torres, E.; Yezers'ka, L.; Rost, A.; Calderín, M.; Edo, C.; Rojano, M.; Said, E.; Jerónimo, P.; Arcila, C.; Serrano, A.; Badillo, J. y Corredoira, L. (2011). Uso de Twitter y Facebook por los medios iberoamericanos. El Profesional de la Información, 20(6), 611-620.https:// doi.org/10.3145/epi.2011.nov.02

García-Galera, M. C.; Del-Hoyo-Hurtado, M. y Fernández-Muñoz, C. (2014). Jóvenes comprometidos en la Red: El papel de las redes sociales en la participación social activa. Comunicar, 22 (43), 35-43.https://doi.org/10.3916/C43-2014-03

Gil-Ramírez, M. ,Gómez-de-Travesedo-Rojas, R. \& Almansa-Martínez, A.(2020). Debate político en YouTube: ¿revitalización o degradación de la deliberación democrática? Profesional de la información, 29(6), e290638.https://doi.org/10.3145/epi.2020.nov.38

Giraldo-Luque, S.; Fernández-García, N. y Pérez-Arce, J. C. (2018). La centralidad temática de la movilización \#NiUnaMenos en Twitter. El profesional de la información, 27(1), 96-105. https://doi.org/10.3145/epi.2018.ene.o9

Gumucio-Rivas, R. L. (29 de noviembre de 2020). La revolución de los "frijoleros" en Guatemala. Clarín. https://www.elclarin.cl/2020/11/29/la-revolucion-de-los-frijoleros-en-guatemala/

Harlow, S. (2012). Social media and social movements: Facebook and an online Guatemalan justice movement that moved offline. New media \& society, 14(2), 225-243. https://doi. org/10.1177/1461444811410408 
Harlow, S. y Harp, D. (2012). Collectiveactiononthe Web: A cross-cultural study of social networkingsites and online and offline activism in theUnitedStates and LatinAmerica. Information, communication\&society, 15(2), 196-216. https://doi.org/10.1080/1369 118X.2011.5914.11

Holt, R. (2004). Dialogue on the Internet: language, civic identity and computer-mediated communication. Praeger.

Igartua, J. J. (2006). Métodos cuantitativos de investigación en comunicación. Bosch.

Kim, Y. y Hsuan-Ting, Ch. (2016). Social media and online political participation: The mediating role of exposure to cross-cutting and like-minded perspectives. Telematics and Informatics, 33(2), 320-330. https://doi.org/10.1016/j.tele.2015.08.008

Kruikemeier, S. y Shehata, A. (2016). News media use and political engagement among adolescents: Ananalysis of virtuous circles using panel data. Political Communica-tion,34(2),221-242. https://doi.org/10.1080/10584609.2016.1174760

Laguardia, L. (28 de noviembre de 2020). \#28N: la crónica en imágenes de la "Revolución de los Frijoleros" que pidió la renuncia de Giammattei. Prensa Libre. https://www.prensalibre.com/guatemala/n28-la-cronica-en-imagenes-de-la-revolucion-de-los-frijolerosque-pidio-la-renuncia-de-giammattei

La Vanguardia (3 de diciembre de 2020). Anuncian manifestaciones el sábado contra Congreso y Gobierno de Guatemala. La Vanguardia. https://www.lavanguardia.com/ politica/20201203/4.9854489506/anuncian-manifestaciones-el-sabado-contra-congreso-y-gobierno-de-guatemala.html

López-Aranguren, E. (2016). El análisis de contenido tradicional. En M. García-Ferrando, F. R.; Alvira Martín, L. E.; Alonso Benito y M. Escobar Mercado (Comps.). El análisis de la realidad social. Métodos y técnicas de investigación (p. 594-616). Madrid: Alianza.

Masías, V. H.; Hecking, T. y Hoppe, U. (2018). Social networking site usage and participation in protest activities in 17 Latin American countries. Telematics and informatics, 35(7), 1809-1831. https://doi.org/10.1016/j.tele.2018.05.010

Matassi, M.y Boczkowski, P. J. (2020). Redes sociales en Iberoamérica. Artículo de revisión. El profesional de la información, 29(1), e290104. https://doi.org/10.3145/epi.2020.ene.04

Méndez, G.-E. (4 de diciembre 2020). OEA muestra preocupación por la corrupción en Guatemala. Soy502. https://www.soy502.com/articulo/oea-emite-comunicado-explica-algunos-hallazgos-guatemala-32419

Mourão, R. R.; Saldaña, M.; McGregor, S. C. y Zeh, A.D. (2016).Support for protests in Latin America: classifications and the role of online networking”. Social Sciences, 5(4), 58. https://doi.org/10.3390/socsci5040058

Salzman, R. (2015a). Exploring social media use and protest participation in Latin America. Journal of Latin American communication research, 5(2), 72-85. http://journal.pubalaic. org/index.php/jlacr/article/view/134

Salzman, R. (2015b). Understanding social media use in Latin America. Palabra clave, 18(3), 842-858. http://dx.doi.org/10.5294/pacla.2015.18.3.9

Sampedro, V. (2011). Redes Sociales. Documento I. Documento de trabajo, 16 de septiembre de 2011.

Sánchez-Duarte, J. M. y Fernández-Romero, D. (2017). Subactivismo feminista y repertorios de acción colectiva digitales: prácticas ciberfeministas en Twitter. El profesional de la información, 26(5), 894-902. https://doi.org/10.3145/epi.2017.sep.11

Santos, D. (28 de noviembre de 2020). Pueblo comelón de frijoles. La Hora. https://lahora. gt/pueblo-comelon-de-frijoles/ 
Shirky, C. (2011). The political power of social media. Foreign affairs,9o(1), 28-41. https:// fam.ag/3wMjKLe

Skoric, M. M.; Poor, N. D.; Liao, Y. y Tang, S. W. H. (2011). Online organization of an offline protest: From social to traditional media and back [ponencia] 44th Hawaii International Conference on System Sciences, p. 1-8. https://doi.org/10.1109/HICSS.2011.330

Smolak, E., López, P., Gómez, R. \& Castillo, A.(2018). Lobby social y redes sociales. El caso de la plataforma Stop Impuestos de Sucesiones. En J. Herrero \& M. Trenta (Coords.), El fin de un modelo de políticas (pp. 1338-1353). Cuadernos Artesanos de Comunicación.

Suau-Gomila, J. (2015). Citizens and online media participation. Attitudes and motivations towards participatory journalism and other online practices in London and Barcelona (PhD thesis, University Ramon Llull). https://www.tdx.cat/handle/10803/289347\#page=1

Tince, D.; Liang, Ch. y He, X. (2017). Social media and internet publicevents. Telematics and Informatics, 34(3), 726-739. https://doi.org/10.1016/j.tele.2016.05.024

Treré, E. y Cargnelutti, D. (2014). Movimientos sociales, redes sociales y Web 2.o: el caso del Movimiento por la Paz con Justicia y Dignidad. Communication \& Society, 27(1), 183-203. https://bit.ly/3wL8yOZ

Tusón-Valls, A. (2002). El análisis de la conversación: entre la estructura y el sentido. Estudios de sociolingüística, 3(1),133-153. https://www.textosenlinea.com.ar/academicos/El\%20 an\%C3\%Alliis\%2ode\%2ola\%20conversacion.pdf

Valenzuela, S. (2013). Unpackingthe use of social media for protest behavior: The roles of information, opinion expression and activism. American behavioral scientist, $57(7)$, 920-942. https://doi.org/10.1177/ooo2764213479375

Valenzuela, S.; Arriagada, A. y Scherman, A. (2012). The social media basis of youth protest behavior: The case of Chile. Journal of communication, 62(2), 299-314. https://doi. org/10.1111/j.1460-2466.2012.01635.X

Valenzuela, S.; Correa, T. y Gil-de-Zúñiga, H. (2018). Ties, likes, and tweets: Using strong and weak ties to explain differences in protest participation across Facebook and Twitter use. Political communication, 35(1), 117-134. https://doi.org/10.1080/10584609.2017.1334726

Van-Dijk, T. A. (1999). Análisis crítico del discurso. Revista austral de ciencias sociales, 30, 203-222. https://doi.org/10.4206/rev.austral.cienc.soc.2016.n30-10

Van-Laer, J. y Van-Aelst, P. (2010). Internet and social movement action repertoires. Information, communication \& society, 13(8), 1146-1171. http://dx.doi. org/10.1080/13691181003628307

We Are Social y Hootsuite (2021). Digital 2021 Local Country Headlines-Guatemala. https:// datareportal.com/reports/digital-2021-guatemala

We Are Social y Hootsuite (2021). Global Digital Overview 2021. https://datareportal.com/ reports/digital-2021-global-overview-report

Wodak, R. (2003). De qué trata el análisis crítico del discurso (ACD). Resumen de su historia, sus conceptos fundamentales y sus desarrollos. En R. Wodak y M. Meyer (Comps). Métodos de análisis crítico del discurso (p. 17-34). Barcelona: Gedisa.

Ziccardi, A. (1998). Gobernabilidad y participación ciudadana en la ciudad capital. UNAM / Miguel Ángel Porrúa. http://ru.iis.sociales.unam.mx/handle/IIS/5650 